\title{
WEAK CONVERGENCE OF STOCHASTIC PROCESSES DEFINED ON SEMI-INFINITE TIME INTERVALS
}

\author{
CHARLES STONE
}

In the standard theorems on weak convergence of stochastic processes, it is invariably assumed that the parameter set is a bounded interval. The object of this paper is to indicate that analogues of these theorems for unbounded intervals are also valid. We shall confine our attention to the results of Skorohod [1], and in particular to those results concerning his $J_{1}$ topology.

Let $E$ be a complete separable metric space with metric $\rho$. We denote by $K$ the space of all $E$-valued functions $x(t), 0 \leqq t<\infty$, which at every point have a limit from the left and are continuous from the right. We define on $K$ the topology $J_{1}$ : a sequence $x_{n}(t)$ is said to be $J_{1}$-convergent to $x(t)$ if there exists a sequence of continuous one-toone mappings $\lambda_{n}(t)$ of the interval $[0, \infty)$ onto itself such that for each $N>0$

$$
\sup _{0 \leqq t \leqq N}\left|\lambda_{n}(t)-t\right| \rightarrow 0 \text { and } \sup _{0 \leqq t \leqq N} \rho\left(x_{n}(t), x\left(\lambda_{n}(t)\right)\right) \rightarrow 0 \text { as } n \rightarrow \infty \text {. }
$$

Note that for continuous $x(t), x_{n}(t)$ converges to $x(t)$ in the $J_{1}$ topology if and only if for each $N>0$

$$
\sup _{0 \leqq t \leqq N} \rho\left(x_{n}(t), x(t)\right) \rightarrow 0 \text { as } n \rightarrow \infty \text {. }
$$

Let $X_{n}(t), n=0,1,2, \cdots$, be stochastic processes whose paths are in $K$. We denote by $C$ the collection of all functionals $f$ on $K$ such that (1) $f\left(X_{n}(\cdot)\right)$ are random variables; and (2) $f$ is continuous in the $J_{1}$ topology almost everywhere with respect to the measure on $K$ corresponding to the process $X_{0}(t)$.

The sequence $X_{n}(t)$ is said to be weakly convergent to $X_{0}(t)$ if for all $f \in C$ the distribution of $f\left(X_{n}(\cdot)\right)$ converges to the distribution of $f\left(X_{0}(\cdot)\right)$ as $n \rightarrow \infty$.

Theorem. The sequence $X_{n}(t)$ is weakly convergent to $X_{0}(t)$ if and only if

(1) the finite dimensional distributions of $X_{n}(t)$ converge weakly to the finite dimensional distributions of $X_{0}(t)$ as $n \rightarrow \infty$; and

(2) for every $\epsilon>0$ and $N>0$

Received by the editors August 1, 1962. 
$\lim _{\substack{n \rightarrow \infty ; \\ c \rightarrow 0}} \operatorname{Pr}\left\{\sup _{\substack{t-c<t_{1}<t_{2}<t+c ; \\ 0 \leq t_{1}<t_{2} \leq N}} \min \left[\rho\left(X_{n}\left(t_{1}\right), X_{n}(t)\right) ; \rho\left(X_{n}(t), X_{n}\left(t_{2}\right)\right)\right]>\epsilon\right\}=0$.

As a direct consequence, if almost all the paths of $X_{0}(t)$ are continuous functions, then the above theorem remains valid if (2) is replaced by the simpler condition that

$\left(2^{\prime}\right)$ for every $\epsilon>0$ and $N>0$

$$
\lim _{n \rightarrow \infty ; c \rightarrow 0} \operatorname{Pr}\left\{\sup _{\left|t_{1}-t_{2}\right| \leqq c ; 0 \leqq t_{1}<t_{1} \leqq N} \rho\left(X_{n}\left(t_{1}\right), X_{n}\left(t_{2}\right)\right)>\epsilon\right\}=0 .
$$

Proof. We construct a new metric space $E^{*}$ with metric $\rho^{*}$ as follows: $E^{*}=\{\theta\} \cup\{(e, t) \mid e \in E$ and $0 \leqq t<1\}$ (here $\theta$ is an abstract element corresponding to $t=1)$;

$$
\begin{aligned}
\rho^{*}\left(\left(e_{1}, t_{1}\right),\left(e_{2}, t_{2}\right)\right) & =\pi^{-1} \min \left(1-t_{1} ; 1-t_{2}\right) \tan ^{-1} \rho\left(e_{1}, e_{2}\right)+\left|t_{1}-t_{2}\right|, \\
\rho^{*}((e, t), \theta) & =\rho^{*}(\theta,(e, t))=1-t, \text { and } p^{*}(\theta, \theta)=0 .
\end{aligned}
$$

It is easy to verify that $E^{*}$ is a complete separable metric space.

We denote by $K^{*}$ the space of all $E^{*}$-valued functions $y(t), 0 \leqq t \leqq 1$, which, at every point have a limit from the left and are continuous from the right, and are such that $y(t)=(e, t)$ for some $e \in E, 0 \leqq t<1$, and $y(1)=\theta$. The functions in $K^{*}$ are all continuous from the left at $t=1$ (this is the point of the above construction). We define on $K^{*}$ the topology $J_{1}^{*}$ : a sequence $y_{n}(t)$ is said to be $J_{1}^{*}$-convergent to $y(t)$ if there exists a sequence of continuous one-to-one mappings $\lambda_{n}^{*}(t)$ of the interval $[0,1]$ onto itself such that

$$
\sup _{0 \leqq t \leqq 1}\left|\lambda_{n}^{*}(t)-t\right| \rightarrow 0 \text { and } \sup _{0 \leqq t \leqq 1} \rho^{*}\left(y_{n}(t)\right), \quad y\left(\lambda_{n}^{*}(t)\right) \rightarrow 0 \quad \text { as } n \rightarrow \infty \text {. }
$$

Let $T$ denote the one-to-one transformation from $K$ onto $K^{*}$ which assigns to the function $x(t)$ in $K$ the function $y(t)$ in $K^{*}$, where $y(t)$ $=(x(\tan (\pi / 2) t), t), 0 \leqq t<1$, and $y(1)=\theta$. Letting $\lambda_{n}(t)$ and $\lambda_{n}^{*}(t)$ be related by

$$
\lambda_{n}^{*}(t)=\frac{2}{\pi} \tan ^{-1} \lambda_{n}\left(\tan \frac{\pi}{2} t\right),
$$

we see that $x_{n}(t)$ converges to $x(t)$ in the $J_{1}$ topology if and only if $y_{n}(t)=T x_{n}(t)$ converges to $y(t)=T x(t)$ in the $J_{1}^{*}$ topology.

Consider the stochastic process $Y_{n}(t)=T X_{n}(t)$. The path functions of $Y_{n}(t)$ are all in $K^{*}$. We denote by $C^{*}$ the collection of all functionals $f^{*}$ on $K^{*}$ such that (1) $f^{*}\left(Y_{n}(\cdot)\right)$ are random variables for $n=0,1,2, \cdots$, and (2) $f^{*}$ is continuous in $J_{1}^{*}$ topology almost everywhere with respect to the measure on $K^{*}$ corresponding to the process $Y_{0}(t)$. 
Conditions (1) and (2) of the above theorem are easily shown to be respectively equivalent to the following conditions:

$\left(1^{*}\right)$ the finite dimensional distributions of $Y_{n}(t)$ converge weakly to the finite dimensional distributions of $Y_{0}(t)$ as $n \rightarrow \infty$; and

$\left(2^{*}\right)$ for every $\epsilon>0$

$\lim _{\substack{n \rightarrow \infty ; \\ c \rightarrow 0}} \operatorname{Pr}\left\{\sup _{\substack{t-c<t_{1}<t<t_{3}<t+c ; \\ 0 \leqq t_{1}<t_{3} \leqslant 1}} \min \left[\rho^{*}\left(Y_{n}\left(t_{1}\right), Y_{n}(t)\right) ; \rho^{*}\left(Y_{n}(t), Y_{n}\left(t_{2}\right)\right)\right]>\epsilon\right\}=0$.

By Theorems 3.2.1-3.2.3 of [1] (see remark below), $\left(1^{*}\right)$ and $\left(2^{*}\right)$ are necessary and sufficient in order that for all $f^{*} \in C^{*}$ the distribution of $f^{*}\left(Y_{n}(\cdot)\right)$ converges to the distribution of $f^{*}\left(Y_{0}(\cdot)\right)$ as $n \rightarrow \infty$. Thus (1) and (2) are necessary and sufficient in order that for all $f \in C$ the distribution of $f\left(X_{n}(\cdot)\right)$ converges to the distribution of $f\left(X_{0}(\cdot)\right)$ as $n \rightarrow \infty$. This completes the proof of the above theorem.

REMARK. In Skorohod's paper $K^{*}$ is replaced by $K_{E^{*}}$, the space of all functions $y(t)$ defined on the interval $[0,1]$ whose values lie in $E^{*}$, and which at every point have a limit from the left and are continuous from the right (and from the left at $t=1$ ). But $K^{*}$ is a measurable subset of $K_{E^{*}}$, and Skorohod's proofs work when $K^{*}$ is substituted for $K_{E^{*}}$.

\section{REFERENCE}

1. A. V. Skorohod, Limit theorems for stochastic processes, Teor. Verojatnost. i Primenen. 1 (1956), 261-290. (Russian)

Cornell University 